\title{
Downregulation of EGFR in a metastatic brain lesion of EGFR-mutated non-small cell lung cancer using a tyrosine kinase inhibitor: A case report
}

\author{
MASATOSHI TAKAGAKI ${ }^{1}$, MANABU KINOSHITA ${ }^{1}$, KAZUMI NISHINO $^{2}$, MASAKAZU NAKANO $^{3}$, \\ HIROKO ADACHI $^{3}$, MORIO UENO ${ }^{4}$, MASANORI KITAMURA ${ }^{5}$, YASUNORI FUJIMOTO ${ }^{6}$, \\ KEI TASHIRO ${ }^{3}$, YASUHIKO TOMITA ${ }^{5}$, FUMIO IMAMURA ${ }^{2}$ and TOSHIKI YOSHIMINE $^{6}$
}

\begin{abstract}
Departments of ${ }^{1}$ Neurosurgery and ${ }^{2}$ Thoracic Oncology, Osaka Medical Center for Cancer and Cardiovascular Diseases, Osaka 537-8511; Departments of ${ }^{3}$ Genomic Medical Sciences and ${ }^{4}$ Ophthalmology, Kyoto Prefectural University of Medicine, Kyoto 606-8507; ${ }^{5}$ Department of Pathology, Osaka Medical Center for Cancer and Cardiovascular Diseases, Osaka 537-8511; ${ }^{6}$ Department of Neurosurgery, Osaka University Graduate School of Medicine, Suita, Osaka 565-0871, Japan
\end{abstract}

Received May 22, 2016; Accepted November 25, 2016

DOI: 10.3892/ol.2017.5677

\begin{abstract}
Brain metastasis is a common complication in patients with cancer, with lung cancer being the most frequent origin of brain metastases. Epidermal growth factor receptor (EGFR) tyrosine kinase inhibitors (TKIs) have begun to serve a pivotal role in lung cancer treatment and have been reported to demonstrate anticancer activity against brain metastases by penetrating the blood-brain barrier. The present study reports, to the best of our knowledge, the first case of EGFR-mutated non-small cell lung cancer (NSCLC) brain metastasis that was surgically resected while the lesion was responding to the EGFR-TKI erlotinib. The results of the present study demonstrated that EGFR-mutated NSCLC cells were able to evade the cytotoxic effect of EGFR-TKI by downregulating EGFR expression, without exhibiting the T790M EGFR mutation.
\end{abstract}

\section{Introduction}

Brain metastasis is a common complication in patients with cancer, with lung cancer being the most frequent origin of brain metastases (1). Lung cancer has an $\sim 40 \%$ chance of metastasizing to the brain during the course of disease progression (1). Current standard treatments for lung cancer brain metastasis patients include surgery, stereotactic radiosurgery and whole-brain radiation therapy (1). It has long been considered that the blood-brain barrier (BBB) hampers the delivery of chemotherapeutic agents to the metastatic brain

Correspondence to: Dr Manabu Kinoshita, Department of Neurosurgery, Osaka Medical Center for Cancer and Cardiovascular Diseases, 1-3-3 Nakamichi, Higashinari-ku, Osaka 537-8511, Japan E-mail: mail@manabukinoshita.com

Key words: epidermal growth factor receptor, tyrosine kinase inhibitor, brain metastasis lesion, and systemic antineoplastic therapy has been reported to demonstrate limited or no efficacy in brain metastases $(2,3)$.

In addition to chemotherapeutic agents, molecular targeting agents, including erlotinib, have begun to serve an important role in the treatment of lung cancer. Erlotinib is an orally active epidermal growth factor receptor (EGFR) tyrosine kinase inhibitor (TKI), which is effective against advanced non-small cell lung cancer (NSCLC) with EGFR mutations. Potentially due to their low molecular mass, EGFR-TKIs are able to cross the $\mathrm{BBB}$ and have been reported to demonstrate anticancer activity against NSCLC brain metastases by penetrating the BBB $(1,2,4)$. However, the underlying mode of action or molecular mechanisms of metastatic brain lesion resistance to EGFR-TKIs remain unclear. Although immunohistological features of resected lung cancer following EGFR-TKI treatment have been reported in an attempt to elucidate the mechanisms of resistance, no immunohistological study of EGFR status has been performed on brain metastasis while still responding to EGFR-TKI. The present study reports, to the best of our knowledge, the first case of EGFR-mutated NSCLC brain metastasis that was surgically resected while the lesion was responding to erlotinib, and aims to elucidate the mode of response of metastatic brain lesions from EGFR-mutated NSCLC to EGFR-TKIs, and investigate the possible underlying mechanisms involved in drug resistance.

\section{Case report}

Case presentation. A 66-year-old Japanese female non-smoker was examined following the complaint of a severe cough. The patient was admitted to Osaka Medical Center for Cancer and Cardiovascular Diseases (Osaka, Japan), a prefectural designated cancer center, in June 2013. Written informed consent was obtained for the analysis described in this report. A chest X-ray revealed a focal mass in the right lung with massive pleural effusion (Fig. 1). Cytodiagnosis of the pleural effusion identified an adenocarcinoma with an L858R EGFR mutation in exon $21 .{ }^{18} \mathrm{~F}$-fluorodeoxyglucose 


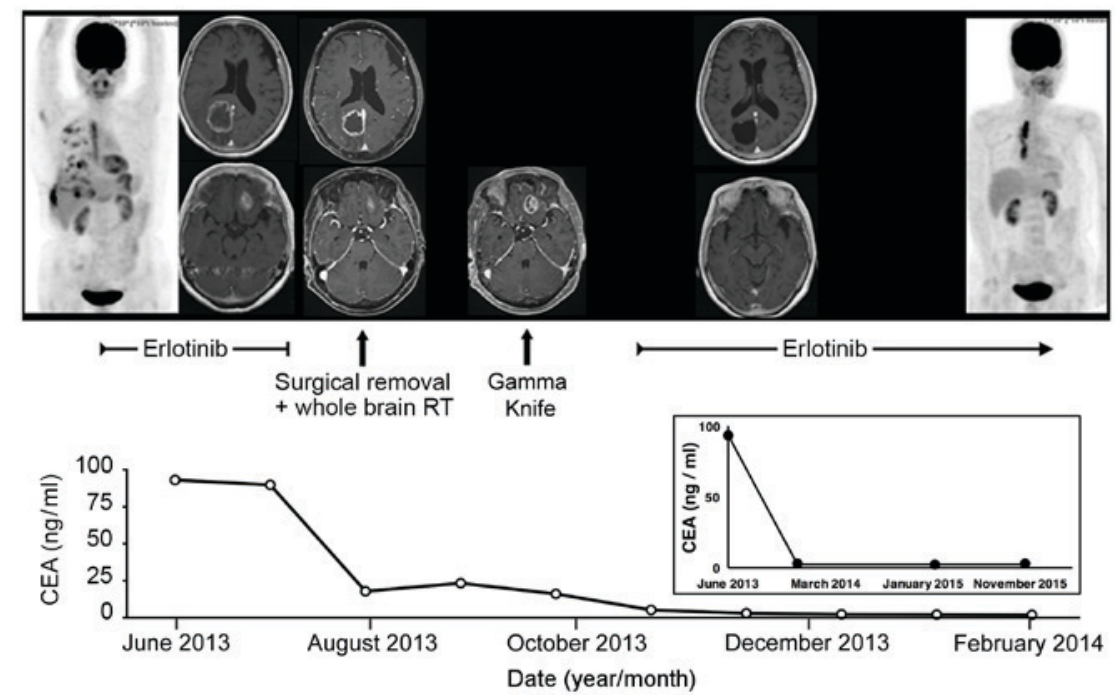

Figure 1.Clinical treatment regime of the case in the present study. The patient was diagnosed with stage IV lung adenocarcinoma (clinical Tumor-Node-Metastasis stage, cT3N1M1b) with an L858R epidermal growth factor receptor mutation in exon 21. Oral administration of erlotinib was initiated during the pre-neurosurgical period in order to restore deteriorated respiratory function. Brain metastasis was surgically resected followed by whole-brain RT and Gamma Knife radiosurgery. Erlotinib maintenance therapy was initiated thereafter. The serum CEA level was maintained within the normal range following initiation of treatment. RT, radiation therapy; CEA, carcinoembryonic antigen.

whole-body positron emission tomography scan revealed brain metastases. Subsequently brain magnetic resonance imaging (MRI) revealed that the lesion had a diameter of $>30 \mathrm{~mm}$ in the right parietal lobe (Figs. 1 and 2A). The patient was diagnosed with stage IV lung cancer [classified using the clinical Tumor-Node-Metastasis staging system 7 th edition (5) as cT3N1M1b].

As severe pleural effusion limited the safety of a neurosurgical procedure aiming to resolve neurological symptoms, including gait disturbance deriving from the metastatic brain lesion, oral administration of erlotinib was initiated during the pre-surgical period in order to restore deteriorated respiratory function. Although erlotinib rapidly regressed pleural effusion and recovered respiratory function, a follow-up MRI 2 weeks following initiation of erlotinib therapy revealed only a slight shrinkage of the brain metastasis (Figs. 1 and 2B). The lesion remained $>30 \mathrm{~mm}$ and the tumor was surgically resected (Fig. 1C), followed by whole-brain radiation therapy with a total dose of $30 \mathrm{~Gy}$. Gamma Knife radiosurgery was performed on the left frontal lobe lesion followed by erlotinib maintenance therapy. The serum carcinoembryonic antigen level was maintained within the normal range for 2.5 years following initiation of treatment with no evidence of radiological recurrence (Fig. 1).

Pathological findings. Surgical tissue was rapidly fixed in $10 \%$ formalin buffer and embedded in paraffin. Extensive necrosis was observed in the tissue with only a slight papillary proliferation at the edge of necrotic tissues (Figs. 2D and 3). Immunohistochemical (IHC) analysis demonstrated that viable cells were positive for thyroid transcription factor 1 and napsin A, confirming that those cells were derived from lung cancer [anti-human thyroid transcription factor 1 (8G7G3/1) and anti-napsin A antibodies; Dako, Agilent Technologies, Inc., Tokyo, Japan; dilution, 1:100; antigen retrieval, microwave heating in $0.1 \mathrm{~mol} / 1$ sodium citrate solution ( $\mathrm{pH} \mathrm{6.0)]} \mathrm{(Fig.} \mathrm{3)} \mathrm{(6).} \mathrm{The} \mathrm{marker} \mathrm{of} \mathrm{prolif-}$ eration Ki-67 labeling index [MIB-1 antibody; dilution 1:20; Immunotech, Marseilles, France; antigen retrieval, microwave heating in $0.1 \mathrm{~mol} / 1$ sodium citrate solution ( $\mathrm{pH} \mathrm{6.0)]}$ was low and few viable cells were Ki-67-positive, whereas strong positivity was observed for endothelial cadherin (E-cadherin) [anti-human E cadherin antibody (NHC-38); Dako, Agilent Technologies, Inc.; dilution 1:100; antigen retrieval, microwave heating in $0.1 \mathrm{~mol} / 1$ sodium citrate solution (pH 6.0)] (Fig. 3).

Subsequently, the EGFR mutation status was evaluated using IHC analysis. Tissues were blocked in goat serum for $1 \mathrm{~h}$ at $37^{\circ} \mathrm{C}$ and incubated with primary antibodies (Cell Signaling Technology Japan, K.K., Tokyo, Japan; dilution, 1:100) at $4^{\circ} \mathrm{C}$ overnight. Antibodies specific for L858R-mutant EGFR (43B2), exon 19-deleted EGFR (E746-A750del; D6B6) and pan-EGFR (D38B1) were used. All specimens were recorded using a Virtual Slide image capturing system (Olympus Corporation, Tokyo, Japan). In contrast with expectations, no EGFR-positive staining was observed in the E-cadherin-positive viable cells (Fig. 3).

Genetic analysis. In addition to IHC examination, genetic examination of the mutation status of the EGFR gene was performed. Fluorescence resonance energy transfer-based preferential homoduplex formation assay (F-PHFA) technology was used as previously described (7). This technique relies on the theory that labeled double-stranded DNA (dsDNA) and a non-labeled amplicon conduct competitive hybridization under a slow thermal gradient. A strand-exchange reaction takes place only when the labeled dsDNA and non-labeled amplicon are $100 \%$ identical in length and sequence, which causes the labeled dsDNA to be diluted by the excess amount of amplicon and emits a fluorescent signal led by the separation of the strand with the fluorescent molecule and the strand with the quenching molecule (7). 


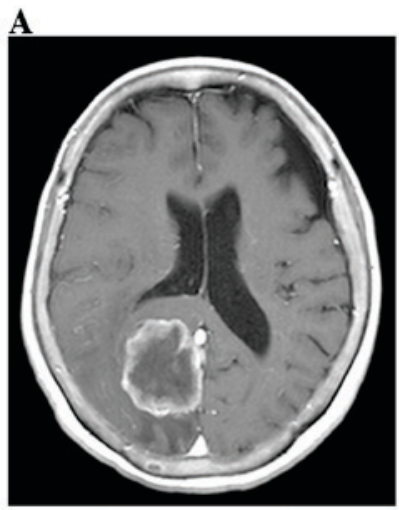

D

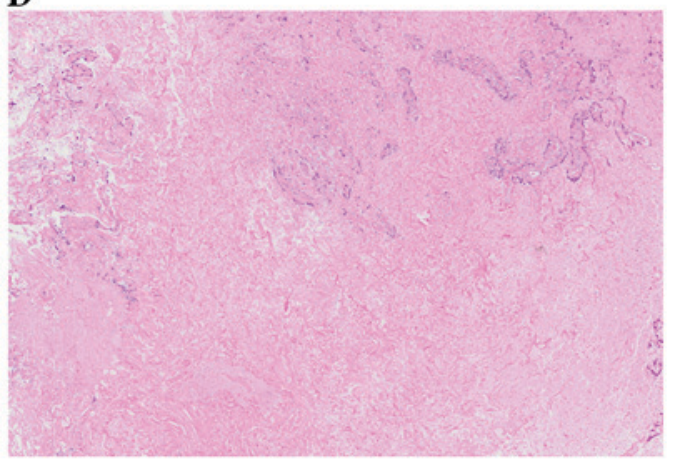

B
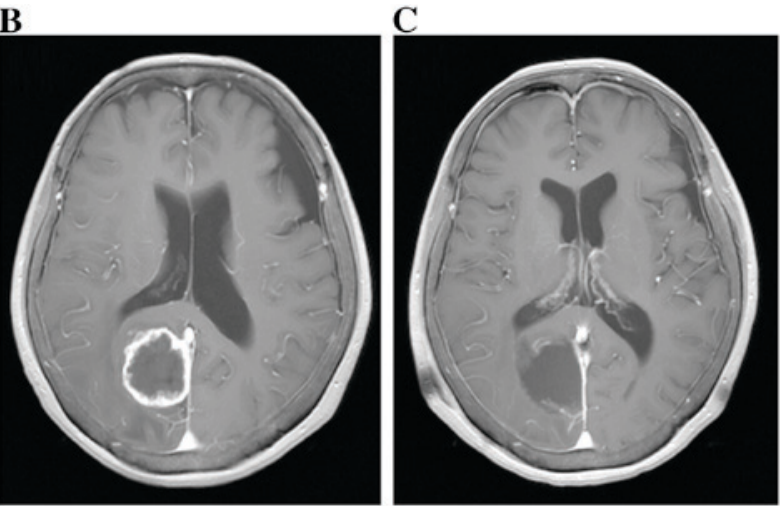

$\mathbf{E}$

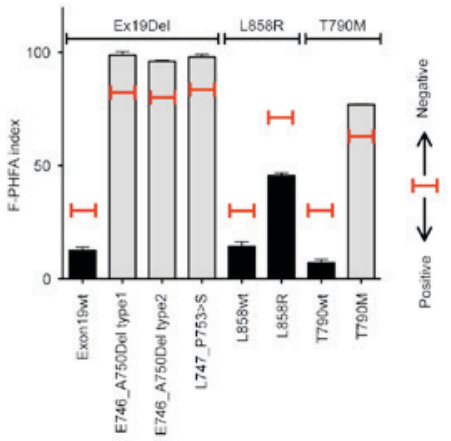

Figure 2. Brain magnetic resonance imaging during the course of treatment and macroscopic view with F-PHFA gene analysis of the removed tissue. Brain magnetic resonance imaging of (A) pretreatment, (B) 2 weeks following erlotinib treatment with marked shrinkage of the tumor and (C) post-surgery achieving gross total resection of the tumor. (D) Necrosis was observed with slight papillary proliferation at the edge of necrotic tissues (magnification, $\mathrm{x} 40$ ). (E) For F-PHFA gene analysis, positive signals are defined by F-PHFA indexes lower than the threshold, indicated by red lines. The EGFR mutation status was Ex19Del-negative, L858R mutation-positive and T790M mutation-negative. F-PHFA, fluorescence resonance energy transfer-based preferential homoduplex formation assay; Ex19Del, exon 19 deletion; wt, wild-type.
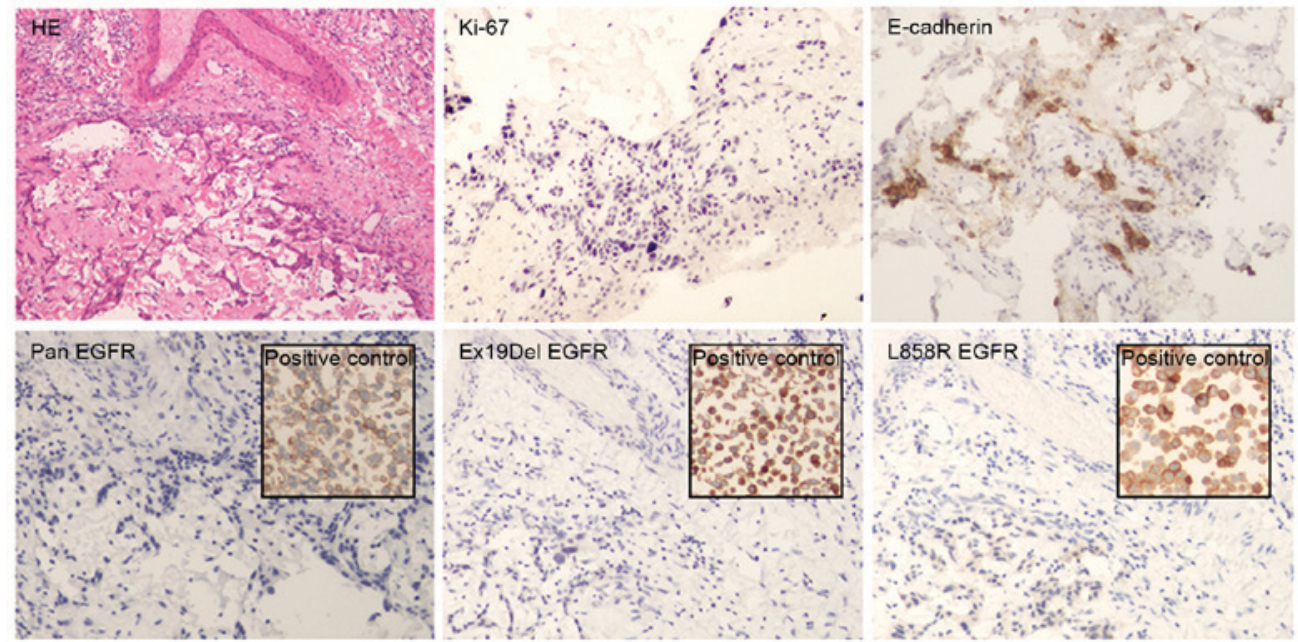

Figure 3. Immunohistochemical staining of the resected tissue. Tissue was stained using HE, Ki-67, E-cadherin, pan-EGFR, exon 19-deleted EGFR and L858R mutant EGFR. Representative images of low Ki-67 labeling index, positive E-cadherin expression and negative EGFR expression. All images are presented at a magnification of x200. HE, hematoxylin and eosin; Ki-67, marker of proliferation Ki-67; E-cadherin, endothelial cadherin; EGFR, epidermal growth factor receptor; Ex19Del, exon 19 deletion.

This assay demonstrated that the tissue possessed the L858R EGFR mutation, whereas exon 19 deletion was not detected. The presence of the T790M EGFR mutation, a secondary EGFR mutation that is considered significant for EGFR-TKI tumor resistance $(8,9)$, was also assessed, revealing that the tissue did not have the T790M mutation (Fig. 2E).

\section{Discussion}

Previous case reports have advocated the efficacy of EGFR-TKI for patients with brain metastasis arising from EGFR-mutated lung cancer $(10,11)$. Berger et al (11) reported that the response rates using EGFR-TKI in brain metastasis 
with EGFR mutation were $<80 \%$, which is markedly high when the expected response rate for conventional treatments such as whole-brain radiation therapy is taken into account. Although the concentration of erlotinib in the cerebrospinal fluid is reported to be decreased compared with that in the plasma (3), a disrupted BBB around the metastatic lesion is considered to lead to an increase in the local drug concentration (2). Consistent with previous studies $(1,12,13)$, pre-operative MRI in the present study demonstrated a slight but notable regression of brain metastasis following initiation of EGFR-TKI therapy (Figs. 1 and 2).

Despite a marked amount of data associated with the clinical response of EGFR-mutated lung cancers to EGFR-TKIs $(11,12)$, there is a lack of evidence of the histopathological changes in cancer tissues following EGFR-TKI treatment. A previous study by Lara-Guerra et al (14) demonstrated the histopathological features of lung cancer tissues treated with gefitinib. In the previous study, EGFR-mutated tumors following EGFR-TKI therapy demonstrated decreased tumor cellularity and a decreased Ki-67 labeling index within the fibrous stroma compared with wild-type EGFR adenocarcinoma tumors. Furthermore, the study described that histological features were associated with the clinical response of the tumor to EGFR-TKI (14). In the present study, viable metastatic cells existed as foci within a large necrotic area. It was also confirmed that those viable cells presented decreased proliferative activity evaluated using Ki-67 labeling similar to the study by Lara-Guerra et al (14). These results confirm that EGFR-TKI demonstrates cytotoxic action against brain metastatic lesions, potentially beyond the BBB.

Furthermore, EGFR mutation status during treatment with EGFR-TKI was evaluated using IHC and F-PHFA gene analysis. IHC demonstrated that viable cells did not express L858R mutant EGFR, whereas the use of F-PHFA demonstrated that these cells did have the L858R EGFR mutation. Although viable cells that are resistant to EGFR-TKI treatment possessed the L858R EGFR mutation, EGFR expression was potentially markedly downregulated in those cells. This model is also supported by the fact that these cells demonstrated negative staining when anti-pan-EGFR antibodies were used for immunostaining. The secondary EGFR mutation T790M, which is observed in $~ 50 \%$ of EGFR-TKI-resistant patients (8), is one of the primary mechanisms that is considered important in the development of EGFR-TKI resistance. The T790M EGFR mutation was also evaluated using F-PHFA, demonstrating that viable cells were negative for the T790M EGFR mutation. It is hypothesized that the T790M mutation occurs during EGFR-TKI therapy and may exist prior to treatment $(11,15)$. EGFR-TKI-resistant brain metastatic lesions have been reported to have the T790M EGFR mutation (16), similar to primary cancer (8). The present study is markedly distinct from previously reported cases in that the tumor was resected and evaluated prior to the lesion demonstrating clinical resistance to EGFR-TKI. The results of the present study demonstrate that viable cells, although possessing reduced proliferative capability, are present while the entire lesion responds to EGFR-TKI. Furthermore, to the best of our knowledge, the present study is the first to provide evidence that EGFR-mutated NSCLC cells are able to evade the cytotoxic effect of EGFR-TKI by downregulating EGFR expression, without exhibiting the T790M EGFR mutation.

\section{References}

1. Shimato S, Mitsudomi T, Kosaka T, Yatabe Y, Wakabayashi T, Mizuno M, Nakahara N, Hatano H, Natsume A, Ishii D and Yoshida J: EGFR mutations in patients with brain metastases from lung cancer: Association with the efficacy of gefitinib. Neuro Oncology 8: 137-144, 2006.

2. Jamal-Hanjani M and Spicer J: Epidermal growth factor receptor tyrosine kinase inhibitors in the treatment of epidermal growth factor receptor-mutant non-small cell lung cancer metastatic to the brain. Clin Cancer Res 18: 938-944, 2012.

3. Caffo M, Barresi V, Caruso G, Cutugno M, La Fata G, Venza M, Alafaci C and Tomasello F: Innovative therapeutic strategies in the treatment of brain metastases. Int J Mol Sci 14: 2135-2174, 2013.

4. Katayama T, Shimizu J, Suda K, Onozato R, Fukui T, Ito S, Hatooka S, Sueda T, Hida T, Yatabe Y and Mitsudomi T: Efficacy of erlotinib for brain and leptomeningeal metastases in patients with lung adenocarcinoma who showed initial good response to gefitinib. J Thorac Oncol 4: 1415-1419, 2009.

5. Goldstraw P, Crowley J, Chansky K, Giroux DJ, Groome PA, Rami-Porta R, Postmus PE, Rusch V and Sobin L; International Association for the Study of Lung Cancer International Staging Committee; Participating Institutions: The IASLC lung cancer staging project: Proposals for the revision of the TNM stage groupings in the forthcoming (seventh) edition of the TNM Classification of malignant tumours. J Thorac Oncol 2: 706-714, 2007.

6. Fatima N, Cohen C, Lawson D and Siddiqui MT: TTF-1 and Napsin A double stain: A useful marker for diagnosing lung adenocarcinoma on fine-needle aspiration cell blocks. Cancer Cytopathol 119: 127-133, 2011.

7. Kitano S, Nakayama M, Yamane A, Tsukahara Y and Amano M: Detection of DNA mutations by fluorescence resonance energy transfer-based preferential homoduplex formation assay. Anal Biochem 408: 197-205, 2011.

8. Hammerman PS, Jänne PA and Johnson BE: Resistance to epidermal growth factor receptor tyrosine kinase inhibitors in non-small cell lung cancer. Clin Cancer Res 15: 7502-7509, 2009.

9. Tartarone A, Lazzari C, Lerose R, Conteduca V, Improta G, Zupa A, Bulotta A, Aieta M and Gregorc V: Mechanisms of resistance to EGFR tyrosine kinase inhibitors gefitinib/erlotinib and to ALK inhibitor crizotinib. Lung Cancer 81: 328-336, 2013.

10. Song Z and Zhang Y: Gefitinib and erlotinib for non-small cell lung cancer patients who fail to respond to radiotherapy for brain metastases. J Clin Neurosci 21: 591-595, 2014.

11. Berger LA, Riesenberg H, Bokemeyer C and Atanackovic D: CNS metastases in non-small-cell lung cancer: Current role of EGFR-TKI therapy and future perspectives. Lung Cancer 80: 242-248, 2013.

12. Namba Y, Kijima T, Yokota S, et al: Gefitinib in patients with brain metastases from non-small-cell lung cancer: review of 15 clinical cases. Clin Lung Cancer. 6: 123-128, 2004.

13. Ruppert AM, Beau-Faller M, Neuville A, Guerin E, Voegeli AC, Mennecier B, Legrain M, Molard A, Jeung MY, Gaub MP, et al: EGFR-TKI and lung adenocarcinoma with CNS relapse: Interest of molecular follow-up. Eur Respir J 33: 436-440, 2008.

14. Lara-Guerra H, Chung CT, Schwock J, Pintilie M, Hwang DM, Leighl NB, Waddell TK and Tsao MS: Histopathological and immunohistochemical features associated with clinical response to neoadjuvant gefitinib therapy in early stage non-small cell lung cancer. Lung Cancer 76: 235-241, 2012.

15. Rosell R, Molina MA, Costa C, Simonetti S, Gimenez-Capitan A, Bertran-Alamillo J, Mayo C, Moran T, Mendez P, Cardenal F, et al: Pretreatment EGFR T790M mutation and BRCA1 mRNA expression in erlotinib-treated advanced non-small-cell lung cancer patients with EGFR mutations. Clin Cancer Res 17: 1160-1168, 2011.

16. Scher KS, Saldivar JS, Fishbein M, Marchevsky A and Reckamp KL: EGFR-mutated lung cancer with T790M-acquired resistance in the brain and histologic transformation in the lung. J Natl Compr Canc Netw 11: 1040-1044, 2013. 